livraisons

d'Histoire

de l'Architecture

\section{Livraisons de l'histoire de l'architecture}

33 | 2017

Histoire du (des ?) patrimoine(s)

\title{
Diagnostic patrimonial et histoire urbaine
}

"Diagnosis of heritage and urban history"

"Denkmaldiagnose und Stadtgeschichte"

Loïc Vadelorge

\section{OpenEdition}

\section{Journals}

Édition électronique

URL : http://journals.openedition.org/lha/754

DOI : 10.4000/lha.754

ISSN : 1960-5994

Éditeur

Association Livraisons d'histoire de l'architecture - LHA

Édition imprimée

Date de publication : 15 juin 2017

Pagination : 87-94

ISSN : $1627-4970$

\section{Référence électronique}

Loïc Vadelorge, " Diagnostic patrimonial et histoire urbaine ", Livraisons de l'histoire de l'architecture [En ligne], 33 | 2017, mis en ligne le 16 juin 2019, consulté le 16 octobre 2019. URL : http:// journals.openedition.org//ha/754 ; DOI : 10.4000//ha.754 


\section{DIAGNOSTIC PATRIMONIAL ET HISTOIRE URBAINE}

En consacrant la notion de "sites patrimoniaux remarquables", la loi du 8 juillet 2016 confirme la pertinence d'une législation sur le patrimoine urbain. Si le terme initialement prévu de "cité historique " a été finalement retiré du texte, la question de l'utilisation de l'histoire dans le procès de patrimonialisation de la ville contemporaine est ouvertement posée :

«Sont classés au titre des sites patrimoniaux remarquables les villes, villages ou quartiers dont la conservation, la restauration, la réhabilitation ou la mise en valeur présente, au point de vue historique, architectural, archéologique, artistique ou paysager, un intérêt public ${ }^{1}$."

Le mot « histoire » revient trois fois dans la loi et l'adjectif « historique » quatrevingt-fois, sans que soit réellement précisée de quelle histoire il s'agit, à l'exception d'une référence à "l'histoire de la nation", à propos des domaines nationaux ${ }^{2}$.

Ce recours à l'histoire pour dire ce qui fait patrimoine n'est certes pas neuf. Sous la III République déjà ${ }^{3}$, on votait une loi « sur la conservation des monuments et objets d'art ayant un intérêt historique et artistique " en distinguant explicitement le "point de vue de l'histoire ou de l'art ${ }^{4}$ ». La même formule était reproduite dans la loi du 31 décembre 1913 sur les monuments historiques et ajoutée à la législation sur les sites naturels à protéger " au point de vue artistique, historique, scientifique, légendaire ou pittoresque " par la loi du 2 mai $1930^{5}$. À rebours de cette banalisation de la référence à l'histoire dans ce qui deviendra à la fin du XXe siècle le champ du patrimoine, la loi Cornudet du 14 mars 1919, souvent considérée comme l'origine du code de l'urbanisme mobilisait d'autres notions: «aménagement ", " extension ", " embellissements ", quand bien même elle s'appliquait aussi

1. Loi 2016-925, publiée au JORF du 8 juillet 2016, relative à la création, à l'architecture et au patrimoine, art. 75, titre III, L. 631-1, consulté sur Légifrance.

2. Art. L. 621-34: «Les domaines nationaux sont des ensembles immobiliers présentant un lien exceptionnel avec l'histoire de la Nation et dont l'État est, au moins pour partie, propriétaire. "

3. Arlette Auduc, Quand les monuments construisaient la nation. Le service des monuments historiques de 1830 à 1940, préface de Jean-Michel Leniaud, Paris, La Documentation française, Comité d'histoire du ministère de la Culture, 2008.

4. Loi du 30 mars 1887, titre premier, chapitre premier, article premier, cité dans Poirrier, Philippe (Éd.), Les Politiques culturelles en France, Paris, La Documentation française, retour aux textes, 2002, p. 87.

5. Loi du 31 décembre 1913, chapitre premier, article premier et loi du 2 mai 1930, titre II, art. 4, ibid., p. 91 et 98. 
aux agglomérations à caractère artistique, historique ou pittoresque ${ }^{6}$. De là à déduire l'existence d'une opposition structurelle entre les deux cultures de la ville, celle des protecteurs et celle des aménageurs, il n'y a qu'un pas qu'on se gardera bien de franchir. Les recherches sur les métiers, les pratiques, l'enseignement ont démontré l'importance de l'utilisation de l'histoire dans la gestation du champ professionnel de l'urbanisme'.

Dès 1970 d'ailleurs, dans un article important ${ }^{8}$, Françoise Choay suggérait que "le recours à l'histoire doit permettre de dépasser le conflit entre progressistes et culturalistes ». Partant d'une généalogie des usages de l'histoire par les théoriciens de l'urbanisme dont elle critiquait la "relégation dédaigneuse " (de Fourier à Archigram) ou "l'annexion abusive" (de Sitte à Lefebvre), elle incitait à utiliser l'histoire des villes dans la perspective de leur aménagement selon trois modalités complémentaires : "Faire, par différence, saisir la spécificité de l'espace que nous sommes en droit d'exiger des aménageurs actuels "; " renouveler la notion de fonction, héritée du XIX ${ }^{e}$ siècle et dans laquelle s'engluent aujourd'hui une part des aménageurs »; révéler « les structures archéologiques " de l'espace habité pour résoudre "la déficience sémantique qui affecte les agglomérations nouvelles ». Derrière le procès sous-jacent de l'urbanisme des Zup de l'époque et la fascination de l'auteure pour les différentes voies du structuralisme (anthropologie, archéologie du savoir, linguistique) qui peuvent aujourd'hui sembler datés, la perspective ouverte dans cet article pionnier sur le potentiel d'un usage urbanistique de l'histoire demeure d'actualité.

Reste que Françoise Choay restreint dans ses ouvrages ultérieurs la notion d'histoire aux théories de l'urbanisme qui de son point de vue sont à l'origine de l'invention de la notion de "patrimoine urbain ${ }^{9}$ ", concept intrinsèquement lié, comme elle l'a démontré, à la tension inhérente entre passé et présent dans la production urbaine. Il apparaît cependant que l'histoire, convoquée aujourd'hui pour caractériser le patrimoine urbain ne peut pas se limiter à l'éclairage des conceptions ou des formes urbaines. L'objet de cet article est d'examiner les conditions d'une mobilisation d'une histoire urbaine élargie ${ }^{10}$ au service d'un renouvellement des villes contraint depuis la loi SRU à prendre en compte les « ensembles urbains remar-

6. Viviane Claude, Pierre-Yves Saunier, "L'urbanisme au début du siècle. De la réforme urbaine à la compétence technique", Vingtième Siècle, revue d'histoire, 64, octobre-décembre 1999. p. 25-40.

7. Viviane Claude, Faire la ville. Les métiers de l'urbanisme au XXe siècle, Marseille, Parenthèses, 2006 ; Corinne Jaquand, "De l'usage de l'histoire pour le projet urbain », dans Xavier Guillot (dir.), Ville, territoire, paysage. Vers un nouveau cycle de pensée du projet, Saint-Étienne, Publications de l'Université de Saint-Étienne, 2016, p. 92-101.

8. Françoise Choay, "L'histoire et la méthode en urbanisme ", Annales, Économies, Sociétés, Civilisations, 4, 1970, p. 1143-1154.

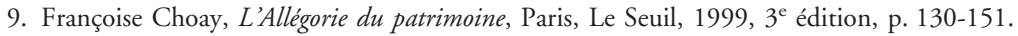

10. Nous défendons ici une conception pluridisciplinaire de l'histoire, en œuvre dans la revue Histoire urbaine ainsi que dans le groupe transversal du Labex Futurs urbains de Paris-Est «Usages de l'histoire et devenirs urbains " : est considérée comme histoire toute discipline travaillant le passé des villes à partir d'archives : histoire de l'architecture et de l'urbanisme, géographie historique, histoire urbaine, etc. 
quables ${ }^{11}$ ". Il s'agit à la fois d'interroger les interactions entre recherche fondamentale et recherche appliquée et de montrer comment les problématiques d'aménagement actuelles suggèrent de compléter les méthodologies du diagnostic patrimonial par le recours plus systématique à l'histoire urbaine ${ }^{12}$.

\section{Histoire et diagnostic urbain}

Le rapprochement souhaitable entre histoire urbaine et diagnostic urbain ${ }^{13}$ se heurte de toute évidence à une contradiction scientifique. Les temporalités de production de l'étude historique d'une ville sont pour de très nombreux chercheurs, incompatibles avec celles de l'urbanisme. L'intelligence historique d'une ville - Marseille, Caen, Rouen ${ }^{14}$ - relève de dizaines d'années de fréquentation de ses archives. Elle est affaire de vies de chercheur ou de programmes collectifs dont la collection " Histoire des villes » dirigée par le médiéviste Philippe Wolff chez Privat dans les années 1970-1980 reste un modèle. Ces sommes académiques s'avèrent peu transposables dans les méthodologies de diagnostic des aménageurs contemporains dont certains font sciemment l'impasse sur l'histoire ${ }^{15}$. L'émergence récente d'un débat autour du contenu et de la pertinence de la notion de « diagnostic patrimonial » au sein de l'Inventaire ${ }^{16}$ atteste que la frontière entre histoire et diagnostic concerne non seulement les deux champs de l'urbanisme et du patrimoine mais aussi la recherche appliquée. Questionner cette frontière nécessite de la mettre en perspective historique.

À première vue, l'injonction à mettre l'histoire au service de l'aménagement constitue une demande sociale récente. Si l'on se préoccupe en France des tissus urbains anciens depuis la loi sur les abords des monuments historiques (25 février 1943 ) et surtout celle des secteurs sauvegardés (4 août 1962), c'est en réalité à la

11. Loi du 13 décembre 2000 relative à la Solidarité et au renouvellement urbain, art. 121-1, alinéa 3, consulté sur Légifrance.

12. Arlette Auduc, "Inventaire général du patrimoine culturel et aménagement ", Les Cahiers de l'IAU $\hat{I} d F, 167$, novembre 2013, p. 27-30.

13. Le terme de diagnostic constitue un mot valise de notre temps. Aucune définition claire n'existe sur cette notion, que ce soit pour son contenu, ses méthodes et sa finalité. Le terme est par conséquent souvent précisé par l'ajout d'un adjectif " patrimonial ", " territorial ", " urbain ", " environnemental ", " immobilier ", etc. qui ne contribue que partiellement à le clarifier. Dans le cadre de cet article le diagnostic urbain implique une dimension historique qui ne limite pas au patrimoine.

14. Marcel Roncayolo, Les Grammaires d'une ville. Essai sur la genèse des structures urbaines à Marseille, Éd. de l'École des hautes études en sciences sociales, 1996 ; Jean-Claude Perrot, Genèse d'une ville moderne, Caen au XVIII siècle, Paris, La Haye-Mouton, 1975 ; Jean-Pierre Chaline, Rouen. Intelligence d'une ville, Rennes, Ouest-France, 2003.

15. À titre d'exemple, Sylvie Lardon, Vincent Piveteau, "Méthodologie de diagnostic pour le projet de territoire : une approche par les modèles spatiaux ", Géocarrefour, 2005, 80-2, p. 75-90.

16. Région Bretagne/Direction du tourisme et du Patrimoine/Service inventaire général du patrimoine culturel. La Nouvelle démarche d'inventaire du patrimoine, 2009, 5 p. ; Les Diagnostics patrimoniaux : des outils pour une politique culturelle du territoire, séminaire de l'Institut national du Patrimoine des 2 et 3 février 2011. 
charnière des années 1990-2000 que la problématique patrimoniale se réactualise et s'ouvre au diagnostic. Les conditions de cette évolution sont connues. La reconversion massive des espaces dédiés à l'industrie modifie en profondeur la gestion courante du foncier. Au temps des réserves foncières programmées (zones d'aménagement différé situées en milieu rural essentiellement) se substitue celui des opportunités disséminées dans l'agglomération (friches industrielles). Le mouvement de décentralisation, qui avait généré ses propres catégories de zones à protéger (loi du 7 janvier 1983 créant les zones de protection du patrimoine architectural, urbain et paysager) est amplifié et contribue à territorialiser le patrimoine, donc à le politiser autrement (de feu les AVAP aux sites patrimoniaux remarquables). Les services patrimoniaux des communes, départements et plus encore des régions (décentralisation de l'Inventaire à partir de la loi du 13 août 2004) sont sollicités pour conforter les projets de territoires. Parallèlement, la crise des banlieues nouvelles engendre une réactivation du renouvellement urbain (loi solidarité et renouvellement urbain du 13 décembre 2000, création de l'ANRU en août 2003) qui ne peut plus passer, comme dans les années 1960 par le bulldozer. Pour refaire la ville sur la ville, il faut désormais dresser des états des lieux répondant à des questions historiques très limitées : où se situent dans l'espace à transformer, les formes architecturales à prendre en compte? Sur quels critères opérer le tri entre conservation et table-rase?

On le comprend, l'injonction au diagnostic génère des pratiques de recherche historique spécifiques. Le relevé de terrain (architectural et paysager) et la production d'outils graphiques (photographies, cartes) tendent la plupart du temps à se substituer au dépouillement des archives et à la construction d'un grand récit de territoire (du quartier à la ville ${ }^{17}$ ). Le paradigme de la méthode régressive qui propose de reconstituer le passé à partir de ses traces actuelles dans l'espace urbain engendre une pratique historique ciblée, qui donne la priorité aux documents les plus accessibles et rentables pour le chercheur (cadastres, plans et cartes). Le passé est trié et mis en valeur par l'image, souvent de grande qualité ${ }^{18}$. La différence entre la recherche historique classique et le diagnostic historique urbain tel que le pratiquent les services régionaux de l'Inventaire ou les CAUE ne tient pas simplement dans l'allègement nécessaire de l'appareil critique ou de la bibliographie. Il faut d'une certaine manière en finir avec le clivage factice entre les mondes universitaires et la recherche patrimoniale appliquée. Les professionnels du patrimoine, formés à l'université, mobilisent les références historiographiques, organisent des colloques ${ }^{19}$ mais leur travail de diagnostic ne consiste pas à questionner le passé ni même à le restituer mais plus simplement à en décrire les qualités, à travers les traces que le chercheur sélectionne dans l'ordinaire urbain.

17. Cf. à titre d'exemple de ces grands récits problématisés : Jean-Louis Robert, Plaisance près Montparnasse, quartier parisien, 1840-1985, Paris, Publications de la Sorbonne, 2012 et Emmanuel Bellanger, Ivry, capitale du communisme municipal, Créaphis, 2017.

18. Cf. à titre d'exemple De Paris à la mer. La ligne de chemin de fer Paris-Rouen-Le Havre, Collection Images du Patrimoine, 2010.

19. Cf. à titre d'exemple Paysages urbains d'Île-de-France. Actes du colloque régional 19-20 septembre 2013, Paris, Somogy, 2014. 
Ce travail de sélection qui est au cœur de la finalité du diagnostic n'a pas été suffisamment interrogé par les travaux qui ont porté sur l'histoire des méthodes de l'urbanisme et du patrimoine. De Françoise Choay (qui a révélé l'ancienneté des méthodes de "surveys " ancêtres des diagnostics urbains) à Nathalie Heinich (qui analyse l'attachement des professionnels de l'Inventaire à la posture de «chercheur " ${ }^{20}$ ) la littérature scientifique s'est surtout intéressée à la production de la valeur historique dans l'enquête urbaine. Or, les usages de l'histoire dans la recherche appliquée relèvent eux-mêmes d'une histoire qui n'est pas simplement celle des conceptions de l'urbanisme ou de l'histoire de l'art mais aussi celle des enjeux et des contextes de l'aménagement. L'histoire de la résorption des îlots insalubres par exemple a mobilisé des formes d'enquêtes sur les immeubles et les tissus urbains qui n'ont pas été conçues et réalisées dans les mêmes conditions entre l'Entre-deux-guerres, la période de Reconstruction et les années $1960^{21}$. Il faudrait par ailleurs restituer les connections oubliées entre les types de diagnostic à une même époque et sur un même objet. Les recherches commanditées (services patrimoniaux, agences d'urbanisme, bureaux d'études, etc.) entrent en concurrence avec des pratiques associatives militantes qui sont parfois très anciennes. La société des Amis des monuments rouennais sauve ainsi en 1900 à l'issue d'une campagne médiatique nationale une vieille maison à pans de bois que les experts municipaux voulaient détruire pour élargir la rue saint-Romain ${ }^{22}$. La saga des secteurs sauvegardés a contribué à produire des diagnostics multiples et divergents dont l'analyse croisée révèle la dimension sociale et politique : ainsi de l'exemple de Tours ${ }^{23}$. Il ne s'agit pas ici d'enfoncer la porte ouverte de l'instrumentalisation ${ }^{24}$ de l'histoire par le diagnostic mais plus simplement de rappeler qu'il relève tout autant de l'espace public que de la tour d'ivoire des chercheurs.

C'est bien l'histoire de la sélection patrimoniale qui mérite aujourd'hui d'être revisitée dans l'intérêt réciproque d'un usage social de la recherche universitaire et de l'enrichissement des questionnements de la recherche appliquée.

20. Nathalie Heinich, La Fabrique du patrimoine. De la cathédrale à la petite cuillère, Paris, éditions MSH, 2009.

21. Isabelle Backouche, Paris transformé. Le Marais 1900-1980: de l'âlot insalubre au secteur sauvegardé, Paris, Creaphis, Lieux Habités, 2016.

22. Loïc Vadelorge, Rouen sous la III République. Politiques et pratiques culturelles, Rennes, PUR, 2005.

23. Patrice Melé, "Habitants mobilisés et devenir d'un espace patrimonial ", Géocarrefour, 2004-79-3, p. 223-230 et Isabelle Backouche, "Expertiser la rénovation urbaine. Le cas de la France dans les années 1960 ", Genèses, 2008-1, p. 45-65.

24. Vincent Veschambre, Traces et mémoires urbaines. Enjeux sociaux de la patrimonialisation et de la démolition, Rennes, PUR, 2008. 


\section{De l'histoire de l'art à l'histoire urbaine}

La fièvre patrimoniale de la fin du $\mathrm{XX}^{\mathrm{e}}$ siècle a contribué à ouvrir un champ des possibles que ni les chercheurs ni les aménageurs n'ont fini d'épuiser ${ }^{25}$. Le contraste est cependant important entre le "tout patrimoine " proclamé et le patrimoine diagnostiqué qui ne concerne qu'1\% des espaces urbains au début du $\mathrm{XXI}^{\mathrm{e}}$ siècle ${ }^{26}$. La grille d'entrée du diagnostic patrimonial reste prioritairement attachée aux centres historiques et privilégie, comme au XIX ${ }^{\mathrm{e}}$ siècle, l'inventaire des bâtiments remarquables soit du fait de leur valeur artistique, soit du fait de leur simple ancienneté dans l'environnement urbain. Le primat octroyé aux monuments et aux vieilles pierres témoigne du poids considérable de l'histoire de l'art dans la formation des chercheurs-producteurs de diagnostic patrimonial. Les buttes témoins que leur œil exercé distingue sont d'abord des œuvres du passé, que la photographie révèle au temps présent. Il ne faut donc pas s'étonner de voir l'auto-critique de services dédiés comme l'Inventaire en passer par une interrogation fondamentale sur le statut de la photographie dans le processus de sélection des paysages urbains. Dans un récent catalogue d'exposition sur "Les origines du Grand Paris ", la cheffe du service Patrimoines et Inventaire du conseil régional Île-de-France souligne l'intérêt du projet "Le Grand Paris vu de nos fenêtres" lancé en 2010 par le Théâtre de la Nuit et qui propose sur le mode collaboratif aux Franciliens de donner à voir leur paysage ordinaire ${ }^{27}$. Du côté de la recherche urbaine, on assiste au même regain d'intérêt pour les clichés d'amateurs, à l'image du programme de recherche portant sur les correspondants de l'Humanité ${ }^{28}$ qui donne à voir une autre mémoire de l'Île-de-France. On l'aura compris, cette mise en avant du regard des habitants ne vise pas tant à confronter le professionnel du diagnostic aux pratiques ordinaires de la ville que de rappeler que la patrimonialisation participe d'un processus social $^{29}$.

À mieux y regarder la déstabilisation du diagnostic patrimonial ne relève pas uniquement de la mise en cause du regard esthète sur le paysage urbain. Le changement d'échelle du renouvellement urbain consécutif aux phénomènes de métropolisation a engendré de nouvelles demandes de diagnostic ${ }^{30}$. La connexion entre les méthodes de l'inventaire architectural et les méthodes de l'urbanisme s'est pour partie opérée au début du XXI siècle via l'injonction à renseigner l'impensable : le

25. Jean-Michel Leniaud, Les Archipels du passé : le patrimoine et son histoire, Fayard, 2002.

26. Régis Neyret, "Du monument historique au tout patrimoine », Géocarrefour, 2004, 79-3, p. 235.

27. Julie Corteville, "Photographier le Grand Paris ", Julie Corteville, Marie-Pierre Deguillaume (dir.), Aux origines du Grand Paris. 130 ans d'histoire. Catalogue de l'exposition du Musée d'histoire sociale de Suresnes, Compiègne, 2016, p. 196-203.

28. Le responsable scientifique en est Thierry Bonzon, maître de conférences à l'UPEM, Cf. http:// acp-regardcollectif.univ-mlv.fr/

29. Caroline de Saint-Pierre (dir.), La Ville patrimoine. Formes, logiques, enjeux et stratégies, Rennes, PUR, 2014.

30. Un bon exemple de cette nouvelle demande est fourni dans Arlette Auduc (dir.), Diagnostic patrimonial de l'OIN Orly-Rungis-Seine-Amont, Paris, Île-de-France, 2014. 
patrimoine des grands ensembles ou des villes nouvelles dont on commençait à détruire des quartiers (de la barre Debussy à la Courneuve en 1986 au quartier de la Croix-Petit de Cergy-Pontoise en 2006 ${ }^{31}$ ) sans en avoir fait l'histoire. Plus encore que pour le remploi du patrimoine industriel ou le repérage des architectures scolaires et universitaires qui ont ces dernières années contribué à conforter la grille de lecture architecturale du diagnostic, les nouveaux ensembles urbains entrés dans la sphère de l'ANRU ont conduit les professionnels du patrimoine à lire les formes architecturales de la seconde moitié du $\mathrm{XX}^{\mathrm{e}}$ siècle (barres, tours, dalles, germes de ville, mégastructures) pour ce qu'elles étaient : des formes urbaines.

Dès lors que ce type de glissement s'opère, on peut augurer de la possibilité de réintroduire la prise en compte des populations dans la production du diagnostic et de suggérer la possibilité d'un diagnostic historique urbain. Retrouver les caractéristiques et les aspirations des pionniers pour rappeler que le bâti a fait sens ${ }^{32}$, décrire les trajectoires résidentielles pour dépasser le cliché de la malédiction des quartiers sensibles ${ }^{33}$ et comprendre le sens et l'évolution des usages sociaux de l'espace urbain forment quelques pistes de ce type de diagnostic ${ }^{34}$. Ce travail, qui se situe à la confluence de la démographie, de la sociologie et de l'ethnologie de la ville contemporaine est encore pour partie devant nous, même si les collaborations des historiens au diagnostic territorial sont désormais bien assumées ${ }^{35}$. Il n'est pas pour autant incongru de penser qu'il est réalisable sur certains espaces urbains qui font l'objet de controverses bloquant leur ré-aménagement. Le concours de l'histoire urbaine à la clarification de ces débats pourra prendre plusieurs formes complémentaires.

L'histoire permet tout d'abord de situer le projet urbain contemporain dans le temps long ${ }^{36}$. Restituer la généalogie des projets permet de dépersonnaliser le débat public contemporain et de comparer dans la durée les options et les blocages de l'aménagement. L'histoire permet ensuite d'articuler les différentes dimensions d'un diagnostic : architectures et formes urbaines, peuplement et activités, culture et politique. Le récit historique autorise les décideurs à se positionner par rapport à un héritage global que le paradigme de la « crise urbaine » contribue à réduire de facto ${ }^{37}$.

31. Lionel Engrand, Olivier Millot (dir.), Cergy-Pontoise. Formes et fonctions d'une ville nouvelle, Paris, Éd. du Pavillon de l'Arsenal, 2015.

32. L'Esprit pionnier ou l'aventure des premiers habitants de la ville nouvelle, Catalogue d'exposition de l'Écomusée de Saint-Quentin-en-Yvelines, 2000.

33. Alexandra Caille, "Les villes nouvelles ont-elles été plus accueillantes pour les étrangers ? L'exemple de Saint-Ouen-l'Aumône (1968-2001)", Loïc Vadelorge (dir.), Habiter les villes nouvelles, Paris, Manuscrit.com, 2006, p. 223-246.

34. Matthieu Giroud, "Usages des espaces rénovés et continuités populaires en centre ancien ", Espaces et Sociétés, 2011-1, p. 37-54.

35. Annie Fourcaut, Emmanuel Bellanger, Mathieu Flonneau, Paris/banlieues Conflits et solidarités, historiographie, anthologie, chronologie, 1788-2006, Paris, Créaphis, 2007 ; Florence Bourillon, Annie Fourcaut (dir.), Agrandir Paris (1860-1970), Paris, Publications de la Sorbonne, 2012.

36. Pauline Rossi, "Constructions et démolitions dans le faubourg Saint-Antoine (1930-1990)", Histoire urbaine, 2015-2, p. 115-135.

37. Danièle Voldman, "Sur les crises urbaines ", Vingtième siècle, revue d'histoire, 1999-64, p. 5-10. 
Il est l'une des conditions de l'association des habitants à la transformation de l'urbain, au-delà des procédures souvent inadéquates de "participation " au cours desquelles la distance sociale entre l'urbaniste ou l'ingénieur et l'usager engendre l'incompréhension et la suspicion. L'histoire permet enfin de questionner les mots valises d'une époque (des "zonages » des Trente Glorieuses aux "banlieues » des années $1980^{38}$, du "cadre de vie " des années 1970 au "pittoresque " du temps présent $^{39}$ ) en démontrant qu'ils forment parfois des symptômes réversibles ${ }^{40}$ ou de simples habits neufs ${ }^{41}$.

Les conditions techniques de la recherche historique ont changé. Si le temps d'imprégnation d'un contexte urbain à étudier reste nécessairement important, l'accessibilité des données, leur mise en réseau et les processus de collaboration propres aux humanités numériques (Gallica, Huma-num, Gertrude, Alpage, Inventer le Grand Paris, etc.) permettent d'envisager une recherche historique appliquée venant compléter les apports reconnus de l'histoire de l'architecture et de l'urbanisme. Le diagnostic patrimonial a pu s'enrichir, depuis une quinzaine d'années du recours à l'histoire des techniques, dans le cadre du long travail de deuil des sociétés industrielles $^{42}$ : il lui reste cependant à mobiliser plus avant les acquis de l'histoire urbaine et plus largement des sciences sociales. Si le législateur a contribué à faire du patrimoine architectural et urbain une ressource et non plus une contrainte de l'aménagement, la rencontre des deux mondes de la recherche historique sur la ville doit révéler le potentiel des sociétés urbaines qui reste en définitive la seule garantie de la transmission durable du passé.

LoÏc VADELORGE professeur d'histoire contemporaine, université Paris-Est Marne-la-Vallée, directeur du laboratoire analyse comparée des pouvoirs, directeur du master diagnostic historique et aménagement urbain

38. Laurent Coudroy de Lille, Christian Topalov, Jean-Charles Depaule, Brigitte Marin (dir.), L'Aventure des mots de la ville, Paris, Robert Laffont, 2010.

39. Voir à titre d'exemple l'étude sur les origines du pittoresque de la Butte Bergeyre à Paris réalisée par Vétillart, Thomas, Densifier l'Est parisien dans l'Entre-deux-guerres : le lotissement des ButtesChaumont (1926-1937), Mémoire de master Diagnostic historique et aménagement urbain, UPEM, 2016, dactyl.

40. Alain Faure, "Un faubourg, des banlieues ou la déclinaison du rejet ", Genèses, 2003-2, p. 48-69.

41. Annie Fourcaut, "Les habits neufs des politiques de la ville depuis 1980 ", Vingtième siècle, revue d'histoire, 1999-64, p. 113-119.

42. Sabine Barles, L'Invention des déchets urbains, France, 1790-1970, Paris, Seyssel-Champ Vallon, 2005. 